\title{
Backstepping for Partially Unknown Nonlinear Systems Using Gaussian Processes
}

\author{
Alexandre Capone and Sandra Hirche
}

\begin{abstract}
In this paper, a backstepping control approach is developed and analyzed for a setting where the system model is partially unknown and is modeled using Gaussian processes. The proposed approach encompasses the classical backstepping and command filtered approaches as special cases. The tracking error is globally uniformly ultimately bounded, and the performance is shown to be improved by adding new training data. The stability analysis is carried out by employing a quadratic Lyapunov function and Tikhonov's theorem. The proposed method outperforms an established adaptive backstepping approach given sufficient training data.
\end{abstract}

Index Terms-Data-driven, Gaussian processes, machine learning, nonlinear control systems, uncertainty

\section{INTRODUCTION}

Backstepping is a widely employed control design approach for trajectory tracking for a wide class of nonlinear systems [1]. In settings where system uncertainties are present, adaptive backstepping approaches mitigate these uncertainties by updating the system model during the control process [2], [3]. However, adaptive backstepping methods require a parametric structure for the model to be assumed a priori, which can lead to poor performance if the choice of parametric structure is inadequate.

In practice, backstepping requires multiple time derivatives of the system models to be computed, which implies a prohibitively complex control law formulation for highdimensional systems. The use of command filters to compute the control signals has become a common form of avoiding such formulations [3]-[5]. Formal guarantees for command filtered backstepping approaches are given in [3], [4]. However, [4] requires exact system knowledge, which is a rigid assumptions in practice, whereas [3] requires a parametric structure for the system uncertainties to be specified a priori. In the context of system identification for control, Gaussian processes (GPs) are increasingly employed [6]-[8]. GPs provide a flexible nonparametric data-driven modelling framework that incorporates an automatic trade-off between data fitting and regularization in noisy settings [9]. Moreover, GP models explicitly quantify system uncertainty, which can be employed to derive model error bounds [10]. Other conventional system identification tools, such as Volterra series [11] and artificial neural networks [12], require an appropriate selection of the model structure in order to provide satisfying results. Furthermore, such methods do not explicitly quantify

The authors are with the Department of Electrical and Computer Engineering, Technical University of Munich, 80333 Munich, Germany (e-mail: alexandre.capone@tum.de; hirche@tum.de). model uncertainty.

In this work, we employ a command filtered backstepping approach to control a system that is learned with GPs, where the training data is collected prior to the control design. The tracking error is reduced with high probability when new training data points are added. We employ a command filter similar to the one given by [4] without needing full knowledge of the system model. The proposed method contrasts with [3] in that the parametric structure of the model uncertainty is not required, and the model is not updated online.

This paper is structured as follows. Section II describes the problem setting, Section III discusses how GPs are employed in this paper. In Section V, the convergence of the proposed method is analyzed. A numerical simulation is contained in Section VI and Section VII provides some concluding remarks.

\section{Problem Setting}

We consider systems in strict feedback-form [1], which are formulated as

$$
\begin{aligned}
\dot{\mathbf{x}}_{i} & =\mathbf{F}_{i}\left(\mathbf{w}_{i}\right)+\mathbf{G}_{i}\left(\mathbf{w}_{i}\right) \mathbf{x}_{i}, \quad i=1, \cdots, h-1 \\
\dot{\mathbf{x}}_{h} & =\mathbf{F}_{h}\left(\mathbf{w}_{h}\right)+\mathbf{G}_{h}\left(\mathbf{w}_{h}\right) \mathbf{u}, \\
\mathbf{x}_{i}(0) & =\mathbf{x}_{i, 0}, \quad i=1, \cdots, h
\end{aligned}
$$

where $\quad \mathbf{x}_{i} \in \mathcal{X}_{i} \subseteq \mathbb{R}^{n}, i=1, \ldots, h \quad$ are the system's states, $\mathbf{u} \in \mathcal{U} \subseteq \mathbb{R}^{n}$ is the vector of control inputs, and $\mathbf{w}_{i}=\left[\mathbf{x}_{1}^{\mathrm{T}}, \cdots, \mathbf{x}_{i}^{\mathrm{T}}\right]^{\mathrm{T}}, i=1, \ldots, h$, denote the concatenations of the state vectors, which are employed for notational simplicity. Similarly, the notation $\mathcal{W}_{i}=\mathcal{X}_{1} \times \mathcal{X}_{2} \times \cdots \times \mathcal{X}_{i}$ is used to denote concatenations of subspaces of the state space. For the sake of brevity, unless stated otherwise, whenever the subscript $i$ is employed, the full set, i.e., $i=1, \ldots, h$, is referred to. The initial conditions are given by $\mathbf{x}_{i, 0} \in \mathcal{X}_{i}$. The nonlinear functions $\mathbf{F}_{i}: \mathcal{W}_{i} \mapsto \mathbf{R}^{n}$ represent unknown system dynamics, whereas the nonlinear functions $\mathbf{G}_{i}: \mathcal{W}_{i} \mapsto \mathbf{R}^{n \times n}$ are assumed to be known. The arguments of the functions $\mathbf{F}_{i}, \mathbf{G}_{i}$, are henceforth omitted. The states $\mathbf{x}_{i}$ are assumed to be available for measurement during the control process. We assume that the functions $\mathbf{F}_{i}$ vanish at the origin, which is a common assumption for systems in strict feedback form [1]. The functions $\mathbf{G}_{i}$ are invertible for all points within the domain of interest. Even though this requirement can seem somewhat restrictive, in practice it can be bypassed by exploiting physical properties of the system [2]. A wide range of systems can be described using the form given above [1]. The goal of this paper is to design a control signal $\mathbf{u}$, such that the state $\mathbf{x}_{1}$ accurately follows a predefined trajectory $\mathbf{x}_{1, d}(t): \mathbb{R}_{+} \mapsto \mathcal{X}_{1}$. In order to perform this task, 
measurements of the time derivative of the states are assumed to be available, with which a GP model can be computed. The system is then controlled using a backstepping approach. For the system at hand, the following assumptions are made.

Assumption 1. The desired trajectory $\mathbf{x}_{1, d}(t)$ and its derivative with respect to time are bounded.

Assumption 2. For every $i \in\{1, \cdots, h\}$, the functions $\mathbf{F}_{i}$ and $\mathbf{G}_{i}$ are bounded and $h-i$ times continuously differentiable.

These assumptions are are not very restrictive and are common for backstepping settings [1]. The following assumption concerns the reproducing kernel Hilbert space (RKHS) norm $\|\cdot\|_{k_{i}}$ of the functions $\mathbf{F}_{i}$. An RKHS is a Hilbert space induced by a symmetric positivedefinite function $\quad k_{i}: \mathcal{W}_{i} \times \mathcal{W}_{i} \mapsto \mathbb{R}, i \in\{1, \cdots, h\}$, called kernel, and contains elements of the form $\sum_{j=1}^{\infty} \alpha_{j} k_{i}\left(\mathbf{w}_{i}^{(j)}, \cdot\right), \alpha_{j} \in \mathbb{R}, \mathbf{w}_{i}^{(j)} \in \mathcal{W}_{i}$. The respective norm $\|\cdot\|_{k_{i}}$ measures smoothness with respect to the corresponding kernel $k_{i}$. Moreover, for many commonly used kernels, such as the squared-exponential or Matérn kernel, functions from the corresponding RKHS space are Lipschitz continuous. For a detailed discussion on RKHSs, the reader is referred to [13].

Assumption 3. For every $i \in\{1, \cdots, h\}$, the function $\mathbf{F}_{i}$ has a bounded RKHS norm with respect to a known kernel $k_{i}$, i.e., $\left\|F_{l, i}\right\|_{k_{i}} \leq B_{F_{i}}<\infty, l=1, \cdots, n$.

Assumption 4. For every $i \in\{1, \cdots, h\}$, the kernel $k_{i}$ is bounded and $h-i$ times continuously differentiable.

Since the kernels $k_{i}$ encode information about $\mathbf{F}_{i}$, Assumption 3 implies that the functions $\mathbf{F}_{i}$ contain the same characteristics as $k_{i}$, which in this case are boundedness and $h-i$ times continuous differentiability, i.e., Assumption 2. Furthermore, a critical aspect of Assumption 3 is that it implies that the kernels $k_{i}$ can adequately approximate the functions $\mathbf{F}_{i}$. Hence, the choice of $k_{i}$ is governed by knowledge of the system at hand. However, this task is simpler than choosing a parametric structure in nonlinear system identification methods, since kernels pose significantly less restrictions than parametric structures. Multiple characteristics can be encoded by composing different kernels. In practice, squared-exponential kernels are often employed, as they have the property that the members of the corresponding RKHS can uniformly approximate continuous functions on compact sets. Kernels that satisfy this characteristic are called universal kernels [14]. Other examples of universal kernels include the Laplacian and spline kernels. An exhaustive review of kernels and respective properties can be found in [9]. For simplicity of exposition, the case where different kernels are used for each component of $\mathbf{F}_{i}$ is not considered. However, the method and analysis provided in this work extend straightforwardly to such cases.

\section{Gaussian Process Model}

A GP, denoted as $\mathcal{G} \mathcal{P}(m, k)$, is fully specified by a mean function $m: \mathcal{W}_{i} \mapsto \mathbb{R}$ and kernel $k_{i}, i \in\{1, \cdots, h\}$. A GP is as a nonparametric regression tool to approximate a nonlinear function $f: \mathcal{W}_{i} \mapsto \mathbb{R}$ using (potentially noisy) system measurements [9]. Here we consider $\sigma$-sub-Gaussian noise as specified in the sequel.

Definition 1. Let $\sigma>0$. A scalar random variable $r$ is said to be $\sigma$-sub-Gaussian [15] if for every $t \in \mathbb{R}$

$$
\mathbb{E}(\exp (t r)) \leq \exp \left(\frac{1}{2} \sigma^{2} t^{2}\right)
$$

holds, where $\mathbb{E}$ denotes the expected value operator.

Examples of such distributions are Gaussian and uniform distributions. We assume a mean $m$ of zero for all GPs, which does not pose any restrictions and is often assumed if no prior system knowledge is available [9]. Given a $\sigma>0$ and $N$ system measurements of the form $y^{(j)}=f\left(\mathbf{w}_{i}^{(j)}\right)+\varepsilon^{(j)}$, where $\varepsilon^{(j)}$ corresponds to $\sigma$-subGaussian noise, and $\mathbf{w}_{i}^{(j)} \in \mathcal{W}_{i}, j=\{1, \cdots, N\}$, the posterior distribution corresponding to $f$ at a point $\mathbf{w}_{i} \in \mathcal{W}_{i}$ is then computed as a normal distribution $\mathcal{N}\left(\mu_{N}\left(\mathbf{w}_{i}\right), \sigma_{N}\left(\mathbf{w}_{i}\right)\right)$ with mean and covariance

$$
\begin{aligned}
& \mu_{N}\left(\mathbf{w}_{i}\right)=\mathbf{k}^{\mathrm{T}}\left(\mathbf{w}_{i}\right)\left(\mathbf{K}+\sigma^{2} \mathbf{I}\right)^{-1} \mathbf{y}_{N} \\
& \sigma_{N}\left(\mathbf{w}_{i}\right)=k\left(\mathbf{w}_{i}, \mathbf{w}_{i}\right)-\mathbf{k}^{\mathrm{T}}\left(\mathbf{w}_{i}\right)\left(\mathbf{K}+\sigma^{2} \mathbf{I}\right)^{-1} \mathbf{k}\left(\mathbf{w}_{i}\right),
\end{aligned}
$$

where $\mathbf{y}_{N}=\left(y^{(1)}, \ldots, y^{(N)}\right)^{\mathrm{T}} \in \mathbb{R}^{N}$ contains the output measurements, $[\mathbf{k}(\cdot)]_{a}=k\left(\mathbf{w}_{i}^{(a)}, \cdot\right)$, and $[\mathbf{K}]_{a b}=k\left(\mathbf{w}_{i}^{(a)}, \mathbf{w}_{i}^{(b)}\right)$. Hence, in order to compute a GP model, the inverse of an $N \times N$ matrix needs to be computed, which corresponds to a computational complexity of order $O\left(N^{3}\right)$. However, this computation only needs to be performed once per GP model. Afterwards, evaluating a GP model essentially corresponds to $N$ evaluations of the kernel function.

Based on [16], we define the maximum information gain $\gamma_{N}^{i}$, which is given by

$$
\gamma_{N}^{i}=\max _{\tilde{\mathbf{w}}_{i}^{(1)}, \cdots, \tilde{\mathbf{w}}_{i}^{(N)} \in \mathcal{W}_{i}} \frac{1}{2} \log \left|\mathbf{I}+\sigma^{-2} \tilde{\mathbf{K}}\right|,
$$

where $[\tilde{\mathbf{K}}]_{a b}=k\left(\tilde{\mathbf{w}}_{i}^{(a)}, \tilde{\mathbf{w}}_{i}^{(b)}\right)$ and $|\cdot|$ is the determinant operator. Intuitively, $\gamma_{N}^{i}$ measures the reduction of uncertainty achievable in a setting where the measurements are taken in the best possible fashion. On a compact set $\mathcal{W}_{i}, \gamma_{N}^{i}$ has sub-linear dependence on $N$ for a multitude of kernels and can efficiently be approximated up to a small constant by employing the approach given in [16]. The following theorem gives a bound for the model error obtained when using a GP trained using noisy measurements, and is due to [10].

Theorem 1. Let $f: \mathcal{W}_{i} \mapsto \mathbb{R}$ be a nonlinear function, $B_{f} \in \mathbb{R}$ a bound for the corresponding RKHS norm w.r.t. $k_{i}$, i.e., $\|f\|_{k_{i}} \leq B_{f}$, and $\delta \in(0,1)$. For all $N \in \mathbb{N}$, define $\beta_{N}=B_{f}+4 \sigma \sqrt{\gamma_{N}^{i}+1+\ln \left(\frac{1}{\delta}\right)}$, where $\gamma_{N}^{i}$ is defined in (5). Then, for all $N \geq 1$ and $\mathbf{w}_{i} \in \mathcal{W}_{i}$, the following holds with probability of at least $1-\delta$.

$$
\left|f\left(\mathbf{w}_{i}\right)-\mu_{N-1}\left(\mathbf{w}_{i}\right)\right| \leq \beta_{N} \sigma_{N-1}\left(\mathbf{w}_{i}\right) .
$$

If $B_{f}$ is not available a priori, a guess-and-doubling strategy can be employed to obtain an estimate [16]. Moreover, $\sigma$ 
can be estimated by sampling the same data point multiple times. Since $\gamma_{N}^{i}$ grows sub-linearly with $N$ on a compact set, the term $\beta_{N}$ grows slowly with $N$ on a compact set. The covariance term $\sigma_{N-1}\left(\mathbf{w}_{i}\right)$ is typically very small next to training data points [9]. Hence, the variance term $\sigma_{N-1}\left(\mathbf{w}_{i}\right)$ can be decreased if the region of interest is sampled densely enough. This is achieved on a compact set [16] by selecting new training data points $\mathbf{w}_{i}^{(N+1)}$ corresponding to highest model uncertainty, i.e.,

$$
\mathbf{w}_{i}^{(N+1)}=\arg \max _{\tilde{\mathbf{w}}_{i} \in \mathcal{W}_{i}} \sigma_{N}\left(\tilde{\mathbf{w}}_{i}\right) .
$$

In Section V, we show that the control law enforces compactness of the portion of the state space explored by the state trajectory.

For each entry of the functions $\mathbf{F}_{i}$, a GP is trained using measurements of the form $y_{l, i}^{(j)}=F_{l, i}\left(\mathbf{w}_{i}^{(j)}\right)+\varepsilon^{(j)}, \mathbf{w}_{i}^{(j)} \in \mathcal{W}_{i}$. The necessary measurements can readily be obtained from measurements of the derivatives $\dot{\mathbf{x}}_{i}^{(j)}$, by subtracting the known components $\mathbf{G}_{i}\left(\mathbf{w}_{i}^{(j)}\right) \mathbf{x}_{i-1}^{(j)}, \ldots, \mathbf{G}_{h}\left(\mathbf{w}_{h}^{(j)}\right) \mathbf{u}^{(j)}$. Even though a multivariate GP formulation exists [9] and can be used to approximate each $\mathbf{F}_{i}$ by a single GP, such a formulation is very cumbersome and corresponds to a high computational cost, as opposed to employing multiple scalar GPs. The estimated models $\hat{\mathbf{F}}_{i}$ are then given by

$$
\hat{\mathbf{F}}_{i}=\left(\begin{array}{lll}
\mu_{1, i}\left(\mathbf{w}_{i}\right) & \cdots & \mu_{n, i}\left(\mathbf{w}_{i}\right)
\end{array}\right)^{\mathrm{T}},
$$

where $\mu_{1, i}\left(\mathbf{w}_{i}\right), \cdots, \mu_{n, i}\left(\mathbf{w}_{i}\right)$ are GP means.

\section{BACKSTEPPING CONTROL WITH GPS}

A backstepping control design approach using GP models is presented. The control law is given directly in the command filtered form, as it encompasses the common backstepping approach as a special case. For a detailed introduction to the standard backstepping method, the reader is referred to [1]. The design approach and notation closely follow that given by [4].

The idea of the command filtered approach is that the control signals $\mathbf{x}_{i, d}, i=2, \cdots, h$ are computed by means of a second order filter with natural frequency $\omega_{f}$, as opposed to employing differentiation. In order to do so, the model estimates $\hat{\mathbf{F}}_{i}$ are firstly computed using data collected prior to the control design. The corresponding formulations are given by (8). The following signals are then defined.

$$
\begin{aligned}
\boldsymbol{\alpha}_{1} & =\mathbf{G}_{1}^{-1}\left(-\hat{\mathbf{F}}_{1}+\dot{\mathbf{x}}_{1, d}-\mathbf{C}_{1} \mathbf{e}_{1}\right) \\
\boldsymbol{\alpha}_{i} & =\mathbf{G}_{i}^{-1}\left(-\hat{\mathbf{F}}_{i}+\dot{\mathbf{x}}_{i, d}-\mathbf{C}_{i} \mathbf{e}_{i}-\mathbf{G}_{i-1} \mathbf{v}_{i-1}\right), \\
i & =2, \cdots, h \\
\mathbf{u} & =\boldsymbol{\alpha}_{h},
\end{aligned}
$$

where $\mathbf{C}_{i} \in \mathbb{R}^{n \times n}$ are positive definite matrices, $\mathbf{v}_{i}=\mathbf{e}_{i}-\boldsymbol{\xi}_{i}$ denotes the difference between the tracking errors $\mathbf{e}_{i}$ and compensation terms $\boldsymbol{\xi}_{i}$, which in turn computed as

$$
\begin{aligned}
\dot{\boldsymbol{\xi}}_{i} & =-\mathbf{C}_{i} \boldsymbol{\xi}_{i}+\mathbf{G}_{i}\left(\mathbf{x}_{i+1, d}-\boldsymbol{\alpha}_{i}\right)+\mathbf{G}_{i} \boldsymbol{\xi}_{i+1}, \\
\dot{\boldsymbol{\xi}}_{i}(0) & =\mathbf{0}, \quad i=1, \cdots, h-1 \\
\boldsymbol{\xi}_{h} & =\mathbf{0}
\end{aligned}
$$

The control signals $\mathbf{x}_{i, d}$ are computed by means of the following second order filter.

$$
\begin{aligned}
\ddot{\mathbf{x}}_{i, d} & =-2 \zeta \omega_{f} \dot{\mathbf{x}}_{i, d}-\omega_{f}^{2}\left(\mathbf{x}_{i, d}-\boldsymbol{\alpha}_{i-1}\right) \\
\mathbf{x}_{i, d}(0) & =\boldsymbol{\alpha}_{i-1}\left(\mathbf{x}_{1}(0), \cdots, \mathbf{x}_{i}(0), \mathbf{x}_{i, d}(0), \mathbf{v}_{i-1}(0)\right) \\
\dot{\mathbf{x}}_{i, d}(0) & =\mathbf{0}, \quad i=2, \cdots, h
\end{aligned}
$$

where $\omega_{f}>0$ and $\zeta \in[0,1)$ are scalar design parameters. By increasing $\omega_{f}$, the difference between $\mathbf{x}_{i, d}$ and $\boldsymbol{\alpha}_{i-1}$ tends to zero more quickly. If $\omega_{f} \rightarrow \infty$ is chosen, then $\mathbf{x}_{i, d}=\boldsymbol{\alpha}_{i-1}$ holds. In this case, one can verify that the control approach corresponds to the standard backstepping equations, with the difference that the exact model dynamics $\mathbf{F}_{i}$ are not known and are substituted by $\hat{\mathbf{F}}_{i}$. The dynamics of the compensation terms $\boldsymbol{\xi}_{i}$ are directly affected by the differences between $\mathbf{x}_{i, d}$ and $\boldsymbol{\alpha}_{i-1}$, and become zero if $\omega_{f} \rightarrow \infty$ holds.

The tracking error dynamics are given by

$$
\begin{aligned}
\dot{\mathbf{e}}_{1}= & \mathbf{F}_{1}-\hat{\mathbf{F}}_{1}-\mathbf{C}_{1} \mathbf{e}_{1}+\mathbf{G}_{1}\left(\mathbf{x}_{2, d}-\boldsymbol{\alpha}_{1}\right)+\mathbf{G}_{1} \mathbf{e}_{2} \\
\dot{\mathbf{e}}_{i}= & \mathbf{F}_{i}-\hat{\mathbf{F}}_{i}-\mathbf{C}_{i} \mathbf{e}_{i}-\mathbf{G}_{i-1} \mathbf{v}_{i-1}+\mathbf{G}_{i}\left(\mathbf{x}_{i+1, d}\right. \\
& \left.-\boldsymbol{\alpha}_{i}\right)+\mathbf{G}_{i} \mathbf{e}_{i+1}, \quad i=2, \cdots, h-1, \\
\dot{\mathbf{e}}_{h}= & \mathbf{F}_{h}-\hat{\mathbf{F}}_{h}-\mathbf{C}_{h} \mathbf{e}_{h}-\mathbf{G}_{h-1} \mathbf{v}_{h-1},
\end{aligned}
$$

whereas the compensated tracking errors are given by

$$
\begin{aligned}
\dot{\mathbf{v}}_{1}= & \mathbf{F}_{1}-\hat{\mathbf{F}}_{1}-\mathbf{C}_{1} \mathbf{v}_{1}+\mathbf{G}_{1} \mathbf{v}_{2} \\
\dot{\mathbf{v}}_{i}= & \mathbf{F}_{i}-\hat{\mathbf{F}}_{i}-\mathbf{C}_{i} \mathbf{v}_{i}-\mathbf{G}_{i-1} \mathbf{v}_{i-1}+\mathbf{G}_{i} \mathbf{v}_{i+1}, \\
& i=2, \cdots, h-1 \\
\dot{\mathbf{v}}_{h}= & \mathbf{F}_{h}-\hat{\mathbf{F}}_{h}-\mathbf{C}_{h} \mathbf{v}_{h}-\mathbf{G}_{h-1} \mathbf{v}_{h-1} .
\end{aligned}
$$

\section{Convergence Analysis}

The tracking error performance resulting from the proposed control law is shown to be directly related to the training data points. Moreover, the performance is improved by adding new training data points. The performance and convergence of the closed loop dynamics are analyzed as follows. Firstly, the control law is analyzed in a setting where $\omega_{f} \rightarrow \infty$ holds, which corresponds to the standard backstepping approach with GP models. Afterwards, the setting where $\omega_{f}<\infty$ holds is analyzed. In order to avoid cumbersome notation, the analysis is performed for a system with $n=1$. However, the analysis can readily be extended to systems where $n>1$ using a similar approach and matrix operations.

For the analysis, the vector

$$
e=\left(e_{1} \cdots e_{h}\right)^{\mathrm{T}}
$$

is used to denote the concatenation of the tracking errors.

\section{A. Convergence Analysis for $\omega_{f} \rightarrow \infty$}

In this section, the convergence of the controlled system for $\omega_{f} \rightarrow \infty$ is analyzed. This corresponds to the standard backstepping design approach [4], with the difference that the system functions $\mathbf{F}_{i}$ are substituted by GP models. The following results show how the tracking error performance depends on the data points used to train the GP models. 
Lemma 1. For each $i=1, \ldots, h$ and $N \in \mathbb{N}$, choose $\beta_{N, h}^{i}=B_{F_{i}}+4 \sigma \sqrt{\gamma_{N}^{i}+1+\ln \left(\frac{1}{\delta_{h}}\right)}$, where $B_{F_{i}} \geq\left\|F_{i}\right\|_{k_{i}}$, $\gamma_{N}^{i}$ is given by (5), and $\delta_{h}=\frac{\delta}{h}$ with $\delta \in(0,1)$. The following then holds with probability of at least $1-\delta$.

$$
\left|F_{i}-\mu_{i}\right| \leq \beta_{N, h}^{i} \sigma_{N}^{i} \quad \forall i=1, \ldots, h, N \in \mathbb{N} .
$$

Proof: Due to Assumption 3 and Theorem 1, $P\left\{\left|F_{i}-\mu_{i}\right|>\beta_{N, h}^{i} \sigma_{N}^{i}\right\}<\delta_{h}$ holds with probability of at least $1-\delta$ for all $i=1, \ldots, h$. Applying the union bound yields

$$
P\left\{\bigcup_{i=1}\left|F_{i}-\mu_{i}\right|>\beta_{N, h}^{i} \sigma_{N}^{i}\right\} \leq \sum_{i=1}^{h} \delta_{h}=h \delta_{h}=\delta .
$$

This in turn implies

$$
P\left\{\bigcap_{i=1}\left|F_{i}-\mu_{i}\right| \leq \beta_{N, h}^{i} \sigma_{N}^{i}\right\} \geq \delta .
$$

Lemma 2. Consider the system given by (1), with $n=1$, and the control law given by (11) with $\omega_{f} \rightarrow \infty$. The system's tracking error $e$ is globally uniformly ultimately bounded. Moreover, with probability of at least $1-\delta$, the system's ultimate error bound is given by

$$
\lim _{t \rightarrow \infty}|\boldsymbol{e}| \leq \frac{\left(\sum_{i=1}^{h}\left(\max _{\mathbf{w}_{i} \in \mathcal{W}_{i}} \beta_{N, h}^{i} \sigma_{N}^{i}\right)^{2}\right)^{\frac{1}{2}}}{\min _{i}\left(C_{i}\right)},
$$

where $\beta_{N, h}^{i}$ is chosen as in Proposition 1,

Proof: For $\omega_{f} \rightarrow \infty$, the differential equations corresponding to the fictitious control signals are reduced to

$$
\begin{aligned}
x_{i+1, d} & =\alpha_{i} \\
\xi_{i} & =-C_{i} \xi_{i}=0 \\
v_{i} & =e_{i}
\end{aligned}
$$

where (28) is due to $\xi_{i}(0)=0$. As such, only the true error dynamics corresponding to the terms $e_{i}$ have to be considered. Equations (18) to (20) can then be reformulated as

$$
\dot{e}=\Delta \boldsymbol{F}-(C+G) e,
$$

where

$$
\begin{aligned}
\Delta \boldsymbol{F} & =\left(\left(F_{1}-\hat{F}_{1}\right) \cdots\left(F_{h}-\hat{F}_{h}\right)\right)^{\mathrm{T}} \\
\boldsymbol{C} & =\operatorname{diag}\left(C_{1}\right. \\
\cdots & \left.C_{h}\right) \\
\boldsymbol{G} & =\left(\begin{array}{ccccc}
0 & G_{1} & 0 & \cdots & 0 \\
-G_{1}^{\mathrm{T}} & 0 & G_{2} & & \vdots \\
0 & -G_{2} & 0 & & \\
\vdots & & & \ddots & G_{h-1} \\
0 & \cdots & & -G_{h-1} & 0
\end{array}\right) .
\end{aligned}
$$

Consider the Lyapunov candidate

$$
V(\boldsymbol{e})=\frac{1}{2} \boldsymbol{e}^{\mathrm{T}} \boldsymbol{e} .
$$

Differentiation with respect to time yields

$$
\begin{aligned}
\dot{V}(\boldsymbol{e})= & \frac{1}{2}\left(\boldsymbol{e}^{\mathrm{T}} \dot{\boldsymbol{e}}+\dot{\boldsymbol{e}}^{\mathrm{T}} \boldsymbol{e}\right)=\sum_{i=1}^{h}\left(e_{i}\left(F_{i}-\hat{F}_{i}\right)-e_{i} C_{i} e_{i}\right) \\
& +\sum_{i=1}^{h-1} e_{i} G_{i+1} e_{i+1}+\sum_{i=2}^{h}\left(-e_{i} G_{i} e_{i-1}\right) \\
= & \boldsymbol{e}^{\mathrm{T}} \Delta \boldsymbol{F}-\boldsymbol{e}^{\mathrm{T}} \boldsymbol{C} \boldsymbol{e} .
\end{aligned}
$$

Hence, $\dot{V}(\boldsymbol{e})<0$ holds if $|\Delta \boldsymbol{F}|<|\boldsymbol{e}| \min _{i}\left(C_{i}\right)$. Due to Assumptions 2 and $4,|\Delta \boldsymbol{F}|$ is bounded. Hence, $|\Delta \boldsymbol{F}|<|\boldsymbol{e}| \min _{i}\left(C_{i}\right)$ holds for $|\boldsymbol{e}|$ large enough, which means that $e$ is globally uniformly ultimately bounded. Lemma 1 in turn implies that $|\Delta \boldsymbol{F}| \leq\left(\sum_{i=1}^{h}\left(\max _{\mathbf{w}_{i}} \beta_{N, h}^{i} \sigma_{N}^{i}\right)^{2}\right)^{\frac{1}{2}}$ holds with probability of at least $1-\delta$, which yields the desired result.

Note that Assumption 3 is not needed to show global uniform ultimate boundedness of $e$. The proof shows that the ultimate error bound of the controlled system can be made arbitrarily small with high probability either by increasing the control gains $C_{i}$ or by selecting data points such that the terms $\sigma_{N}^{i} \beta_{N, h}^{i}$ are reduced. The latter is achieved by collecting new system measurements at points of high uncertainty. This is particularly interesting in a setting where the control input $u$ is constrained and high gains $C_{i}$ cannot be realized. Moreover, the proof shows the control law can be used to enforce compactness of the portion of the state space explored by the state trajectory. This in turn can be employed to guarantee sublinear dependence of $\gamma_{N}^{i}$ on $N$, which enables the ultimate bound of $e$ to be efficiently reduced with new training data.

\section{B. Convergence Analysis for $\omega_{f}<\infty$}

The following section analyzes the performance of the command filtered control, i.e., eqs. (9) to (12) and (14) to (23), for a fixed $\omega_{f}<\infty$.

Lemma 3. The variables $v_{i}$ of the controlled system using (11) are globally uniformly ultimately bounded.

Proof: Consider the Lyapunov candidate

$$
V\left(v_{1}, \cdots, v_{h}\right)=\frac{1}{2} \sum_{i=1}^{h} v_{i}^{2} .
$$

Taking the derivative with respect to time yields

$$
\begin{aligned}
& \dot{V}\left(v_{1}, \cdots, v_{h}\right)=\sum_{i=1}^{h} v_{i} \dot{v}_{i} \\
= & \sum_{i=1}^{h} v_{i}\left(F_{i}-\hat{F}_{i}-C_{i} v_{i}\right)+\sum_{i=2}^{h} v_{i}\left(-G_{i-1} v_{i-1}\right) \\
& +\sum_{i=1}^{h-1} v_{i}\left(G_{i} v_{i+1}\right)=\sum_{i=1}^{h} v_{i}\left(F_{i}-\hat{F}_{i}-C_{i} v_{i}\right) .
\end{aligned}
$$

Hence, $\dot{V}\left(v_{1}, \cdots, v_{h}\right)<0$ if $|\Delta \boldsymbol{F}||\boldsymbol{C}|^{-1}<|\boldsymbol{v}|$. Due to Assumptions 2 and 4 , the difference between $F_{i}$ and $\hat{F}_{i}$ is bounded for $i=1, \ldots, h$, which completes the proof.

The following definition is due to [17, Def. 10.1]. 
Definition 2. A function $y(t, \epsilon)$ is said to be of order $\mathcal{O}(\epsilon)$ if there exist constants $c$ and $k$, such that

$$
|y(t, \epsilon)| \leq k|\epsilon| \quad \forall \epsilon, \quad|\epsilon| \leq c .
$$

This is denoted by $y(t, \epsilon)=\mathcal{O}(\epsilon)$.

Theorem 2. Denote the tracking error and the desired control trajectories obtained using eqs. (9) to (12) and (14) to (23) with $\omega_{f} \rightarrow \infty$, i.e., the solution resulting from the backstepping approach, as $\boldsymbol{e}^{\infty}(t)$ and $x_{i, d}^{\infty}(t)$, respectively. Let $\boldsymbol{e}(t)$ and $x_{i, d}(t)$ be the tracking error and desired control trajectories obtained using eqs. (9) to (12) and (14) to (23) for a fixed $\omega_{f}<\infty$. Choose an arbitrary positive scalar $T>0$. For all $0<t<T$, the following holds.

(i) $\boldsymbol{e}(t)-\boldsymbol{e}^{\infty}(t)=\mathcal{O}\left(\frac{1}{\omega_{f}}\right)$

(ii) $x_{i, d}(t)-x_{i, d}^{\infty}(t)=\mathcal{O}\left(\frac{1}{\omega_{f}}\right)$

(iii) $\frac{1}{\omega_{f}} \dot{x}_{i, d}(t)=\mathcal{O}\left(\frac{1}{\omega_{f}}\right)$.

Proof: The proof consists of showing that all conditions required for Tikhonov's theorem [17, Theorem 11.1] are satisfied by the controlled system. Hence, the notation closely follows that of [17, Theorem 11.1]. In the following proof, the definitions $D_{\mathbf{X}}=: \mathbb{R}^{(2 h-1) n}$ and $D_{\mathbf{Z}}=\mathbb{R}^{(2 h-2) n}$ are utilized. For the proof, the pseudo control signals are redefined as $z_{i, 1}:=x_{i+1, d}$ and $z_{i, 2}:=\frac{1}{\omega_{f}} \dot{x}_{i+1, d}, i=1, \cdots, h-1$. The equations corresponding to the command filter, i.e., eqs. (15) to (17), are thus reformulated as

$$
\begin{aligned}
\dot{z}_{i, 1} & =\omega_{f} z_{i, 2} \\
\dot{z}_{i, 2} & =-2 \zeta \omega_{f} z_{i, 2}-\omega_{f}\left(z_{i, 1}-\alpha_{i}\right) \\
z_{i, 1}(0) & =\alpha_{i}(0) \\
z_{i, 2}(0) & =0 .
\end{aligned}
$$

This enables a similar analysis to the one given in [4]. Define the vectors

$$
\begin{aligned}
\mathbf{X} & =\left[e_{1}, \cdots, e_{h}, \xi_{1}^{\mathrm{T}}, \cdots, \xi_{h-1}^{\mathrm{T}}\right]^{\mathrm{T}} \in D_{\mathbf{X}} \\
\mathbf{Z} & =\left[z_{1,1}, z_{1,2} \cdots, z_{h-1,1}, z_{h-1,2}\right]^{\mathrm{T}} \in D_{\mathbf{Z}}
\end{aligned}
$$

Equations (9) to (12) and (14) to (20) are reformulated as

$$
\begin{aligned}
\dot{\mathbf{X}} & =\mathcal{F}(t, \mathbf{X}, \mathbf{Z}, \epsilon) \\
\epsilon \dot{\mathbf{Z}} & =\mathcal{G}(t, \mathbf{X}, \mathbf{Z}, \epsilon) \\
\mathbf{X}(0) & =\mathbf{X}_{0} \\
\mathbf{Z}(0) & =\mathbf{Z}_{0}
\end{aligned}
$$

where the definition $\epsilon:=\frac{1}{\omega_{f}}$ is utilized. Here the entries of $\mathcal{F}$ correspond to the differential equations for the variables $e_{i}$ and $\xi_{i}, i=1, \cdots, h$, i.e., eqs. (18) to (20) and eqs. (12) and (14). On the other hand, the entries of $\mathcal{G}$ correspond to eqs. (36) and (37) after division by $\omega_{f}$, i.e.,

$$
\begin{aligned}
\mathcal{G}= & {\left[z_{1,2}^{\mathrm{T}} \quad\left(-2 \zeta z_{1,2}-\left(z_{1,1}-\alpha_{1}\right)\right)^{\mathrm{T}}\right.} \\
& \left.\cdots z_{h-1,2}^{\mathrm{T}} \quad\left(-2 \zeta z_{h-1,2}-\left(z_{h-1,1}-\alpha_{h-1}\right)\right)^{\mathrm{T}}\right] .
\end{aligned}
$$

We now show that all three conditions required for [17, Theorem 11.1] hold.
- The functions $\mathcal{F}, \mathcal{G}$, and the initial conditions $\mathbf{X}_{0}, \mathbf{Z}_{0}$ are independent of $\epsilon$. Together with Assumption 1 and Assumption 2, this implies that the following holds on any compact subset of $D_{\mathbf{X}} \times D_{\mathbf{Z}}$. The functions $\mathcal{F}$ and $\mathcal{G}$, their first partial derivatives with respect to $(\mathbf{X}, \mathbf{Z}, \epsilon)$, and the partial derivative of $\mathcal{G}$ with respect to $t$ are continuous and bounded. For $\epsilon=0$, (43) reduces to an algebraic equation whose unique solution is given by

$$
\mathbf{Z}^{\infty}(t)=\left(\begin{array}{lllll}
x_{i, d}^{\infty}(t) & 0 & \cdots & x_{h, d}^{\infty}(t) & 0
\end{array}\right),
$$

and has continuous partial derivatives with respect to $x_{i, d}^{\infty}$ and $t$. Moreover, the Jacobian $\frac{\partial \mathcal{G}(t, \mathbf{X}, \mathbf{Z}, 0)}{\partial \mathbf{Z}}$ has continuous first partial derivatives with respect to its arguments, and the initial conditions $\mathbf{X}_{0}, \mathbf{Z}_{0}$ are smooth functions of $\epsilon$.

- Let $\mathbf{X}^{\infty}(t)$ denote the solution of (42) for $\epsilon=0$. Denote the solution of eqs. (9) to (12) and (14) to (23) for $\omega_{f} \rightarrow \infty$ as $\boldsymbol{x}^{\infty}(t)$. Due to Lemma 1 and Assumption 1, $\boldsymbol{x}^{\infty}(t)$ is globally ultimately uniformly bounded, which implies $\boldsymbol{x}^{\infty}(t) \in \mathcal{S} \subset D_{\mathbf{X}}$ for a compact $\mathcal{S}$. Together with Assumptions 1 and 2, and the Picard-Lindelof theorem [18], this implies that $\boldsymbol{x}^{\infty}(t)$ is unique for $t \in[0, T]$.

- An exhaustive proof of the satisfaction of the third condition is given in [4].

Therefore, there exists a positive constant $\epsilon^{*}$, such that eqs. (42) to (45) possess a unique solution $\mathbf{X}^{\omega_{f}}(t), \mathbf{Z}^{\omega_{f}}(t)$, and the following holds for all $t \in[0, T)$ and all $\epsilon \in\left[0, \epsilon^{*}\right]$.

$$
\begin{aligned}
& \mathbf{X}^{\omega_{f}}(t)-\mathbf{X}^{\infty}(t)=\mathcal{O}(\epsilon) \\
& \mathbf{Z}^{\omega_{f}}(t)-\mathbf{Z}^{\infty}(t)=\mathcal{O}(\epsilon) .
\end{aligned}
$$

Theorem 2 shows that the solution of the controlled system can be brought arbitrarily close to that of the standard backstepping algorithm over an arbitrary finite time interval by increasing the frequency $\omega_{f}$ of the command filter. As such, the system's performance over any finite time interval depends essentially on the quality and number of training data.

Corollary 1. Choose $\beta_{N, h}^{i}$ and $\delta$ as in Lemma 1. For the solution of the controlled system, the following holds for all $t \in[0, T]$ with probability of at least $1-\delta$.

$$
x_{1}(t)-x_{1, d}(t)=\mathcal{O}\left(\frac{1}{\omega_{f}}+\frac{\left(\sum_{i=1}^{h}\left(\max _{\mathbf{w}_{i} \in \mathcal{W}_{i}} \beta_{N, h}^{i} \sigma_{N}^{i}\right)^{2}\right)^{\frac{1}{2}}}{\min _{i}\left(C_{i}\right)}\right)
$$

Proof: The proof follows directly from Theorem 2 and Lemma 1.

\section{Numerical Simulation}

The proposed approach is illustrated for the control a onelink manipulator in a numerical simulation. The simulation results are compared to those obtained using an adaptive neural network-based backstepping method, which is implemented as described in [2]. Note that the approach from [2] makes use of Assumptions 1 and 2, but not Assumptions 3 and 4. However, 
while Assumption 4 is very mild, Assumption 3 is only needed to improve tracking performance, but not to guarantee global uniform ultimate boundedness, as shown in Section V-A.

A one-link manipulator can be described as in (1) by employing the following definitions [2].

$$
\begin{array}{ll}
F_{1}=0, & G_{1}=1 \\
F_{2}=\left(-p_{1} \sin \left(x_{1}\right)-p_{2} x_{2}\right) / p_{3}, & G_{2}=\frac{1}{p_{3}}, \\
F_{3}=\left(-p_{4} x_{2}-p_{5} x_{3}\right) / p_{6}, & G_{3}=\frac{1}{p_{6}},
\end{array}
$$

ultimately limited by the choice of parametric structure, even though it updates its parameters during the control process.

TABLE I

$L_{2}$-NORM OF TRACKING ERRORS OVER TIME INTERVAL $[0,100]$.

\begin{tabular}{|c||c|c|c|}
\hline & Bs. with GPs, $N=5$ & Bs. with GPs, $N=50$ & Adaptive bs. [2] \\
\hline$\|\boldsymbol{e}\|_{L_{2}}$ & 333.2 & 31.4 & 74.8 \\
\hline
\end{tabular}

\section{CONCLUSION}

We present a Gaussian process based backstepping method for systems with partially unknown dynamics. The resulting tracking error is globally uniformly ultimately bounded, and its ultimate bound is decreased by using additional training data. The proposed approach is also employable with command filters, which can be tuned such that the resulting trajectories are arbitrarily close to those achieved using the standard backstepping approach with high probability over a finite time interval. Given enough training data, the proposed method achieves better results than a state-of-the-art adaptive method.

\section{REFERENCES}

[1] M. Krstic, I. Kanellakopoulos, and P. V. Kokotovic, Nonlinear and adaptive control design. Wiley, 1995.

[2] C. Kwan and F. L. Lewis, "Robust backstepping control of nonlinear systems using neural networks," IEEE Trans. on Systems, Man, and Cybernetics-Part A: Systems and Humans, vol. 30, no. 6, pp. 753-766, 2000.

[3] W. Dong, J. A. Farrell, M. M. Polycarpou, V. Djapic, and M. Sharma, "Command filtered adaptive backstepping," IEEE Trans. on Control Systems Technology, vol. 20, no. 3, pp. 566-580, 2012.

[4] J. A. Farrell, M. Polycarpou, M. Sharma, and W. Dong, "Command filtered backstepping," IEEE Trans. on Automatic Control, vol. 54, no. 6, pp. 1391-1395, 2009.

[5] Z. Zuo, "Trajectory tracking control design with command-filtered compensation for a quadrotor,' IET control theory \& applications, vol. 4, no. 11, pp. 2343-2355, 2010.

[6] J. Umlauft, T. Beckers, M. Kimmel, and S. Hirche, "Feedback linearization using Gaussian processes," in IEEE Conference on Decision and Control, 2017. IEEE, 2017, pp. 5249-5255.

[7] J. Umlauft, L. Pöhler, and S. Hirche, "An uncertainty-based control Lyapunov approach for control-affine systems modeled by Gaussian process," IEEE Control Systems Letters, vol. 2, no. 3, pp. 483-488, July 2018.

[8] G. Chowdhary, H. A. Kingravi, J. P. How, P. A. Vela et al., "Bayesian nonparametric adaptive control using Gaussian processes."

[9] C. E. Rasmussen and C. K. Williams, "Gaussian processes for machine learning. 2006," The MIT Press, Cambridge, MA, USA, vol. 38, pp. 715-719, 2006

[10] S. R. Chowdhury and A. Gopalan, "On kernelized multi-armed bandits," in International Conference on Machine Learning, vol. 70. PMLR, 2017, pp. 844-853.

[11] F. Doyle, R. Pearson, and B. Ogunnaike, Identification and Control using Volterra Models. Springer, 2002.

Fig. 1. Tracking errors of system variables for proposed control approach with $N=5$ data points per GP, $N=50$ data points per GP, and alternative adaptive backstepping approach from [2].

expected from Corollary 1, the tracking error converges to a neighborhood of the origin in both cases. When using $N=5$ data points, the long-term performance is poor compared to the adaptive method, as indicated by the high $L_{2}$-norm of the error. By employing $N=50$ data points, the uncertainty in the GP model is reduced, which leads to a lower $L_{2}$-norm of the error than in the adaptive case. This illustrates that the performance of the proposed approach depends on the number and quality of the system measurements, whereas the adaptive method is
[12] L. Ljung, Ed., System Identification (2Nd Ed.): Theory for the User. Upper Saddle River, NJ, USA: Prentice Hall PTR, 1999.

[13] G. Wahba, Spline Models for Observational Data. Philadelphia, PA: SIAM, 1990.

[14] I. Steinwart and A. Christmann, Support vector machines. Springer Science \& Business Media, 2008.

[15] V. V. Buldygin and Y. V. Kozachenko, "Sub-Gaussian random variables," Ukrainian Mathematical Journal, vol. 32, no. 6, pp. 483-489, 1980.

[16] N. Srinivas, A. Krause, S. M. Kakade, and M. W. Seeger, "Informationtheoretic regret bounds for Gaussian process optimization in the bandit setting," IEEE Trans. on Information Theory, vol. 58, no. 5, pp. 3250$3265,2012$.

[17] H. K. Khalil, Nonlinear systems; 3rd ed. Upper Saddle River, NJ: Prentice-Hall, 2002.

[18] E. A. Coddington and N. Levinson, Theory of ordinary differential equations. Tata McGraw-Hill Education, 1955. 